Wu, T., Wong, S. K. H., Law, B. T. T., Grieve, E. , Wu, O., Tong, D. K. H., Leung, D. K. W., Lam, C. L. K. and Wong, C. K. H. (2020) Five-year effectiveness of bariatric surgery on disease remission, weight loss, and changes of metabolic parameters in obese patients with type 2 diabetes: A population-based propensity score-matched cohort study. Diabetes/Metabolism Research and Reviews, 36(3), e3236.

There may be differences between this version and the published version. You are advised to consult the publisher's version if you wish to cite from it.

This is the peer reviewed version of the following article:

Wu, T., Wong, S. K. H., Law, B. T. T., Grieve, E., Wu, O., Tong, D. K. H., Leung, D. K. W., Lam, C. L. K. and Wong, C. K. H. (2020) Five-year effectiveness of bariatric surgery on disease remission, weight loss, and changes of metabolic parameters in obese patients with type 2 diabetes: A population-based propensity score-matched cohort study. Diabetes/Metabolism Research and Reviews, 36(3), e3236. (doi: $\underline{10.1002 / \mathrm{dmrr} .3236)}$

This article may be used for non-commercial purposes in accordance with Wiley Terms and Conditions for Self-Archiving.

http://eprints.gla.ac.uk/207704/

Deposited on: 16 January 2020 


\section{Manuscript no.: DMRR-19-RES-373R1}

Five-year effectiveness of bariatric surgery on disease remission, weight loss and changes of metabolic parameters in obese patients with type 2 diabetes mellitus: A population-based propensity score-matched cohort study

Tingting Wu ${ }^{1}$, Simon Kin Hung Wong ${ }^{2}$, Betty Tsz Ting Law ${ }^{3}$, Eleanor Grieve ${ }^{4}$, Olivia Wu ${ }^{4}$, Daniel King Hung Tong ${ }^{5}$, David Kai Wing Leung ${ }^{6}$, Cindy Lo Kuen Lam ${ }^{1}$, Carlos King Ho Wong ${ }^{1}$

${ }^{1}$ Department of Family Medicine and Primary Care, The University of Hong Kong, Hong Kong SAR, China

${ }^{2}$ Department of Surgery, Prince of Wales Hospital, Hong Kong SAR, China

${ }^{3}$ Division of Esophageal and Upper Gastrointestinal Surgery, Department of Surgery, The University of Hong Kong, Hong Kong SAR, China

${ }^{4}$ Health Economics and Health Technology Assessment, Institute of Health \& Well-being, University of Glasgow, UK

${ }^{5}$ Surgery Centre, Hong Kong Sanatorium \& Hospital, Hong Kong SAR, China

${ }^{6}$ Department of Surgery, United Christian Hospital, Hong Kong SAR, China

Short running title: 5-year effect of bariatric surgery

Word count (abstract): 249

Word count (main body): 4485

Number of references, tables, and figures: $53,1,3$

Corresponding Author: Carlos K.H. Wong, Department of Family Medicine and Primary Care, The University of Hong Kong. Address: Rm 1-01, 1/F, Jockey Club Building for Interdisciplinary Research, 5 Sassoon Road, Pokfulam, Hong Kong. Tel: (+852) 2831-5055; Fax: (+852) 2814-7475; Email: carlosho@hku.hk 


\begin{abstract}
:
Aims: To compare disease remission rates, weight loss and changes of metabolic parameters of patients after bariatric surgery with non-surgical patients.

Methods: Based on 2006-2017 Hospital Authority database, a population-based retrospective cohort of obese type 2 diabetes mellitus (T2DM) patients with and without bariatric surgery were identified. Surgical patients were matched with non-surgical patients on 1-to-5 propensity score. Remission rates of diabetes, hypertension and dyslipidaemia were reported annually up to 60 months. Changes in weight loss measurements (Body Mass Index [BMI], percentage of total weight loss $[\% \mathrm{TWL}]$, percentage of excess weight loss [\%EWL], and percentage of rebound in excess weight loss [\%REWL]) and metabolic parameters (HbA1c, systolic blood pressure [SBP], diastolic blood pressure [DBP] and Low-density lipoprotein cholesterol [LDLC]) were measured for both groups.
\end{abstract}

Results: 401 surgical patients (310 restrictive surgeries; 91 bypass surgeries) and 1,894 nonsurgical patients were included. Surgical patients had higher remission rates in diabetes and dyslipidaemia, and better glycaemic control at 12-60-month (all $\mathrm{p}<0.01$ ). SBP and DBP were significantly lower for surgical group up to 12-month, and similar between two groups after 12-month. Surgical patients had significantly lower BMI during follow-up period. \%TWL and \%EWL were higher in the surgery group (15.7\% vs. 3.7\%; $48.8 \%$ vs. $12.0 \%)$ at 60 -month $(p<0.001)$; differences in \%REWL between two groups were insignificant. The effectiveness of restrictive and bypass surgeries was similar at 60-month, although restrictive surgeries were slightly more effective in T2DM remission.

Conclusions: Bariatric surgery was effective in weight loss, remission of diabetes and dyslipidaemia in 5-year post-surgery.

\title{
Keywords:
}

Bariatric surgery, BMI, Diabetes remission, Obesity, Population-based study, Type 2 Diabetes 


\section{Manuscript}

\section{Introduction}

Bariatric surgery is currently considered as one of the most effective therapies for morbidly obese patients (1), since approximately $30 \%$ of initial weight of patients is reduced (2, 3) and their weight loss is maintained for 10-15 years after the surgery (4). Evidence has also suggested that bariatric surgery can ameliorate metabolic syndromes, as well as help patients achieve diabetes and other metabolic disorders remission (5-7). Around 75-90\% of patients who underwent bariatric surgery achieved comorbidity resolution, particularly for patients with insulin resistance, hypertension and respiratory disorders (4). Though relapse in recovered patients may occur over time, long-term effect of bariatric surgery on disease remission was supported by a systematic review, where $66.7 \%$ of T2DM patients, $38.2 \%$ of hypertensive patients, and $60.4 \%$ dyslipidaemia patients achieved disease remission at up to 5 years after bariatric surgery (8). A prospective cohort study with a follow-up across fifteen years found that diabetes remission rates dropped gradually but still exceeded $30 \%$ at 15 years after the surgery (9).

Bariatric surgery has been recommended as a treatment option of the management of obesity and T2DM in several guidelines (10-12) and gained global popularity since last few decades, due to its safety and effectiveness in obese patients. The total number of bariatric surgery performed worldwide grew rapidly from 146,301 in 2003 to 468,609 in 2013 (13). A similar exponential increase in bariatric surgery performed was also found in Asia Pacific countries, whereby the number almost doubled during the period of 2011-2013 and reached to 46,110 in 2013 (13). In the past few years, many studies affirmed the efficacy of bariatric surgery on weight reduction, disease remission, and changes of disease-related clinical parameters among Chinese and other Asian populations (14-21). However, most of these studies either had a limited follow-up period up to 24 months $(14,17-20)$, or recruited relatively small number of patients from a single centre $(15,16)$.

Whether types of surgery affect the effectiveness of bariatric surgery in T2DM remission and weight reduction has become a hot research topic. All current evidences indicate that laparoscopic sleeve gastrectomy (LSG) and other bariatric surgical procedures, including 
laparoscopic gastric bypass (LGB) and laparoscopic adjustable gastric banding (LAGB), are all well-tolerated and efficacious procedures for obese patients with T2DM (22-25). However, the answer to if one surgical procedure is superior to another is uncertain and still under further investigation.

Therefore, a long-term population-based study using real-world data to estimate the effectiveness of bariatric surgery and to compare different surgical procedures should be conducted. This study aimed 1) to evaluate the effect of bariatric surgery on multiple disease remissions on obese patients with T2DM, 2) to illustrate the changes of metabolic parameters over five years by using population-based data, and 3) to compare the effectiveness between restrictive and bypass surgeries.

\section{Materials and Methods}

\section{Study Design and Patient Sampling}

This study was a population-based retrospective propensity score-matched cohort study. Patients with T2DM undergoing bariatric surgery between 1 January 2006 and 31 December 2017 were retrieved from Hospital Authority Clinical Management System (HA/CMS) by International Classification of Diseases, Ninth Revision, Clinical Modification (ICD-9-CM) procedure Codes. T2DM was identified by ICD-9-CM diagnosis codes and International Classification of Primary Care (ICPC-2) diagnosis codes (Supplemental Table 1). HA is a statutory body that is accountable to Hong Kong government. Indeed, HA/CMS contains valid and substantial clinical data, which have been largely used in population-based studies (26, 27). Patients with type 1 diabetes or gestational diabetes were excluded from this study. In line with International Federation for the Surgery of Obesity and Metabolic Disorders-Asia Pacific Chapter (IFSO-APC) (28) and international guideline and consensus (29), the BMI threshold of $27.5 \mathrm{~kg} / \mathrm{m}^{2}$ was one of the surgery indications for Asian patients. Hence, patients with a BMI reading $\geq 27.5 \mathrm{~kg} / \mathrm{m}^{2}$ on the index date were included. A control group of obese patients (BMI $\geq 30 \mathrm{~kg} / \mathrm{m}^{2}$, defined by World Health Organisation [WHO] (30)) who have ever utilised healthcare services and have not ever underwent bariatric surgery during the above observation period was selected for comparison. The index date for surgical patients was set as the date of bariatric surgery. The index date for patients in the control group was the date of bariatric 
surgery of their matched surgical patients. The follow-up period was from the index date to up to 60 months after the index date.

\section{Outcomes}

Primary outcomes were prevalent remission rates, attrition-adjusted remission rates, cumulative remission rates and attrition-adjusted remission rates (31) of diabetes, hypertension, dyslipidaemia, high Low-density Lipoprotein Cholesterol (LDL-C), low High-density lipoprotein Cholesterol (HDL-C), and high triglycerides of both surgical and non-surgical patients up to 60 months after the index date.

Secondary outcomes were the changes of metabolic parameters, including BMI, Haemoglobin A1c (HbA1c), systolic blood pressure (SBP), diastolic blood pressure (DBP), and LDL-C, as well as weight loss measurements, including percentage of total weight loss (\%TWL), excess weight loss (\%EWL), and percentage of rebound in excess weight loss (\%REWL). As HbA1c $<7.0 \%$ is one of glycaemic goals for adults (32), percentage of patients who achieved this target was also included as one of secondary outcomes. The measurement points of secondary outcomes were index date, within 3 months, 6, 12, 24, 36, 48 and 60 months after the index date.

\section{Definitions}

Complete diabetes remission was defined as normoglycaemia with $\mathrm{HbA} 1 \mathrm{c}<6.0 \%$ and fasting glucose $<5.6 \mathrm{mmol} / \mathrm{L}$ for at least 1 year without any anti-diabetic pharmacologic therapy or ongoing bariatric surgical procedures (33). Partial diabetes remission was defined as HbA1c of 6.0\%-6.5\% and fasting glucose of 5.6-6.9 mmol/L for at least 1 year without any anti-diabetic pharmacologic therapy or ongoing bariatric surgical procedures (33). Remission of hypertension was defined as an SBP $<140 \mathrm{mmHg}$ and a DBP $<90 \mathrm{mmHg}$ for at least 1 year without anti-hypertensive pharmacologic therapy (34). Remission of dyslipidaemia was defined as LDL-C $<4.14 \mathrm{mmol} / \mathrm{L}, \mathrm{HDL}-\mathrm{C} \geq 1.03 \mathrm{mmol} / \mathrm{L}$, and triglycerides $<2.26 \mathrm{mmol} / \mathrm{L}$ without taking any lipid-lowering medication for at least 1 year. Based on the recent American Association of Clinical Endocrinologists and American College of Endocrinology 
(AACE/ACE) dyslipidaemia guideline (35), remission of high LDL-C, low HDL-C and high triglycerides was defined as LDL-C $<2.59 \mathrm{mmol} / \mathrm{L}$ (for moderately risk people), HDL-C >1.03 $\mathrm{mmol} / \mathrm{L}$, and triglycerides $<2.26 \mathrm{mmol} / \mathrm{L}$ for at least 1 year without any lipid-lowering medications, respectively.

For patients who initially experienced disease remission after the index date, relapse of different diseases was defined as follows. Relapse of diabetes was defined as restarting diabetes medications, HbA1c $>6.5 \%$, or fasting glucose $\geq 6.9 \mathrm{mmol} / \mathrm{L}$. Relapse of hypertension was defined as SBP $\geq 140 \mathrm{mmHg}$, DBP $\geq 90 \mathrm{mmHg}$, or retaking any anti-hypertensive drugs. Relapse of dyslipidaemia was defined as LDL-C $\geq 4.14 \mathrm{mmol} / \mathrm{L}$, HDL-C $<1.03 \mathrm{mmol} / \mathrm{L}$, triglycerides $\geq 2.26 \mathrm{mmol} / \mathrm{L}$, or taking a lipid-lowering medication. Relapse of high LDL-C (for moderately risk people) was defined as either restarting lipid-lowering medications or LDL-C $\geq 2.59 \mathrm{mmol} / \mathrm{L}$. Relapse of low HDL-C was defined as either restarting lipid-lowering medications or HDL-C $\leq 1.03 \mathrm{mmol} / \mathrm{L}$. Similarly, relapse of high triglycerides was defined as either restarting lipid-lowering medications or triglycerides $\geq 2.26 \mathrm{mmol} / \mathrm{L}$.

Prevalent remission rate was calculated as dividing the number of patients who were in remission at the time of measurement by the number of patients retained, whereas cumulative remission rates were ratios of number of patients who have ever achieved remission and the number of patients retained. For attrition-adjusted remission rates, extreme-case imputation was adopted, i.e. patients who lost to follow-up were considered as no remission (31). Therefore, attrition-adjusted prevalent remission was calculated as dividing the number of patients who were in remission at the time of measurement by the number of patients with the certain disease at the index date, while attrition-adjusted cumulative remission rates were ratios of number of patients who have ever achieved remission and initial number of patients with the certain disease at the index date (31).

\%TWL was defined as the ratio of weight difference between baseline and postsurgical followups to the baseline weight. \%EWL was calculated as weight difference between baseline and follow-ups divided by the difference between baseline and ideal weight. Ideal weight equalled to the ideal BMI (25 kg/m²) (16) multiplied by square of height in meters. \%REWL was the 
percentage difference between the \%EWL measured and the best \%EWL achieved during the follow-up period (16).

\section{Propensity Score Matching}

To address the absence of baseline data, multiple imputation by chained equations (MICE) (36) was used for both surgical and control patients. The number of imputations was 20. HbA1c, SBP, DBP and LDL-C were imputed by other clinical parameters such as BMI, gender, age, total cholesterol, HDL-C, serum creatinine, triglyceride fasting glucose, Charlson Comorbidity Index, use of oral anti-diabetic drugs, use of insulin, use of anti-hypertensive drugs, use of lipid lowering agents, and history of mental health problems, hyperlipidaemia, obstructive sleep apnoea, gallbladder disease, musculoskeletal and chronic orthopaedic disorders, and hypertension. Model parameters were estimated from multiply imputed data and then used to obtain multiple-imputation linear predictions by applying Rubin's combination rules observation wise to the completed-data predictions (37). Propensity Score Matching was performed using the predictions obtained after MICE.

The propensity scores of all enrolled patients were calculated by using multivariable logistic regression adjusting for baseline covariates. Those covariates included age, gender, BMI, HbA1c, SBP, DBP, total cholesterol, HDL-C, LDL-C, serum creatinine, triglyceride, fasting glucose, Charlson Comorbidity Index, use of oral anti-diabetic drugs, use of insulin, and history of hypertension, mental health problems, hyperlipidaemia, obstructive sleep apnoea, gallbladder disease, musculoskeletal and chronic orthopaedic disorders. The caliper criteria improved the quality of the nearest neighbour matching by specifying a maximum tolerance of the propensity score distance between patients in the surgical group and in the control group. The propensity score matching was performed by 'calipmatch' command on one-to-five basis without replacement in STATA.

\section{Statistical Analysis}

Baseline socio-demographic and clinical characteristics of study population were presented by frequency with percentages for categorical variables and means with standard deviation (SD) 
for continuous variables. Independent t-tests and chi-square tests were used to assess the differences between the surgery and control group for categorical variables and continuous variables, respectively. Covariates balance between the two groups after matching was indicated by standardised mean difference, when the standardised mean differences of baseline covariates were less than 0.1 .

Primary and secondary outcomes at baseline and at follow-ups were displayed in mean values with 95\% confidence interval (CI). Logistic regression models or independent t-tests were used to detect the differences of all these measurements in each follow-up point between two groups and two surgical procedures.

All statistical analyses were performed using the STATA version 13.0 (StataCorp LP, College Station, TX, USA). All significance tests were two-tailed and p-value smaller than 0.05 was considered as statistical significance.

\section{Results}

Population

Supplemental Figure 1 visualises the study flow. A total of 668 patients with relevant ICD9-CM procedure codes were identified. After excluding patients who had index date in the year of 2018, were non-T2DM, had missing BMI, had non-bariatric procedural operation or had BMI $<27.5 \mathrm{~kg} / \mathrm{m}^{2}$ on the index date, 422 patients were included. For patients without bariatric surgery, a total of 288,017 patients were initially identified, and 42,307 eligible patients remained. After 1-to-5 propensity score matching, 401 obese patients in the surgery group were successfully matched with 1,894 obese patients in the control group. Patients were observed for up to 60 months. There were 86 surgical and 394 control patients available for analysis at the end of follow-up. The median follow-up period for 422 surgical patients before matching, 401 patients in the surgery group and 1,894 patients in the control group is 23 months, 23 months and 33 months, respectively. 
Baseline characteristics of study population before and after propensity score matching were summarised in Table 1. For 422 eligible surgical patients before matching, 327 (77.5\%) underwent restrictive surgeries (315 LSG; 12 LAGB) and 95 (22.5\%) underwent bypass surgeries (18 laparoscopic sleeve gastrectomy with duodenojejunal bypass (LSG/DJB); 77 LGB). 1-to-5 propensity score matching method well balanced the socio-demographic background, clinical parameters and comorbidities of surgical and non-surgical patients. The mean BMI of surgical patients and non-surgical patients were $36.81 \mathrm{~kg} / \mathrm{m}^{2}$ and $36.31 \mathrm{~kg} / \mathrm{m}^{2}$ $(\mathrm{p}=0.121)$, respectively; and the T2DM duration of two groups were 4.12 years and 4.36 years $(\mathrm{p}=0.136)$. For 401 obese T2DM patients in the surgery group, 91 (22.7\%) patients underwent bypass procedures (17 LSG/DJB; 74 LGB), and 310 (77.3\%) patients underwent restrictive procedures (299 LSG; 11 LAGB).

\section{Prevalent remission rates}

The diabetes complete remission rates of patients in the bariatric surgery group were significantly higher than those of patients in the control group during the whole follow-up years. The T2DM complete remission rates of surgical patients peaked at 24 months (26.4\%) and fluctuated between $16.7 \%$ and $20.6 \%$ in the remaining years, whereas no more than $3.5 \%$ of patients in the matched control group were free from T2DM over 5 years. (Figure 1a) Prevalent diabetes complete or partial remission rates of surgical patients increased from $5.0 \%$ in 12 months to $28.3 \%$ in 24 months and dropped to $22.1 \%$ in 60 months; while those of non-surgical patients fluctuated slightly around 5.0\% over 60 months. Surgical patients had higher complete or partial diabetes remission rates at and after 24 months $(\mathrm{p}<0.001)$. (Figure 1b)

The prevalent hypertension remission rates of both groups did not exceed $6.5 \%$ during the follow-up period, and bariatric surgery did not show superiority in resolving hypertension when compared with no surgery (p-value ranged from 0.119 to 0.540 ). (Figure 1c) Significantly larger percentage of patients in the surgery group than patients in the matched control group achieved significantly high LDL-C, low HDL-C and high triglycerides remission across 5 years. (Supplemental Figure 3a, 4a, 5a) Bariatric surgery also showed a compelling advantage in resolving dyslipidaemia, as prevalent remission rates of dyslipidaemia of surgical patients were higher than those of non-surgical patients at all measurement points $(\mathrm{p}<0.001)$. (Figure 1d) 


\section{Attrition-adjusted prevalent remission rates}

After adjustment of attrition, prevalent complete diabetes remission rates of surgical patients were still significantly higher than those of non-surgical patients from 12 months to 60 months. (Figure 1e) The patterns of attrition-adjusted prevalent diabetes complete or partial remission rates were similar to those of prevalent diabetes complete or partial remission rates. (Figure 1f) Also, hypertension remission between two groups were not significantly different all the time. (Figure 1g) Similar to prevalent remission rates, attrition-adjusted prevalent remission rates of high LDL-C, low HDL-C, high triglycerides and dyslipidaemia of surgical patients were significantly higher than those of the control group at all measurement points. (Figure 1h; Supplemental Figure 3b, 4b, 5b)

\section{Cumulative remission rates}

Figure 2a-d and Supplemental Figure 3c, 4c and 5c describe the cumulative disease remission rates of both groups. The five-year cumulative diabetes complete remission rates, diabetes complete or partial remission rates, high LDL-C remission rats, low HDL-C remission rates, high triglycerides remission rates and dyslipidaemia remission rates of surgical patients were $25.6 \%$, 32.6\%, 24.3\%, 41.7\%, 38.2\%, and 40.3\%, respectively. All above rates were significantly higher than those of non-surgical patients. However, five-year cumulative rates of hypertension remission did not differ between two groups.

\section{Attrition-adjusted cumulative remission rates}

Similar to the above cumulative remission rates, patients in the surgery group had greater attrition-adjusted cumulative rates in diabetes complete remission, diabetes complete or partial remission, high LDL-C remission, low HDL-C remission, high triglycerides remission, and dyslipidaemia remission over time. Apart from 12 months, when the control group had higher attrition-adjusted cumulative hypertension remission rate $(\mathrm{p}=0.037)$, no other significant differences were found in this outcome from 24 to 60 months. (Figure 2e-h; Supplemental Figure 3d, 4d, 5d). 


\section{Change of metabolic parameters}

The effect of bariatric surgery on HbA1c reduction was significant, as the mean HbA1c of patients in the surgery group dropped rapidly from $7.66 \%$ at baseline to $6.30 \%$ at 6 months, and ended up at $6.93 \%$ at 60 months. The mean HbA1c of surgical patients at each follow-up point was significantly lower than that of their counterparts. Significantly more percentage of T2DM patients in the surgery group achieved the target of HbA1c $<7 \%$ than patients in the control group across 5 years. At 5 year after surgery, the percentage of surgical patients and non-surgical patients achieved this target were $60.0 \%$ and $44.3 \%$, respectively $(\mathrm{p}=0.014)$. Bariatric surgery lowered both SBP and DBP within 12 months, however, the effect did not persist in the remaining years. Non-surgical patients had significantly lower LDL-C level than surgical patients at 6, 36 and 48 months, indicating that bariatric surgery did not show much effect on lowering LDL-C. (Figure 3a-e)

\section{Change of weight Loss measurements}

The mean BMI of patients in the surgery group dropped from $36.81 \mathrm{~kg} / \mathrm{m}^{2}$ at baseline to 32.21 $\mathrm{kg} / \mathrm{m}^{2}$ at 60 months. Although patients in the matched control group also experienced BMI reduction during the follow-up period, their mean BMI was significantly higher than surgical patients on each time point. Furthermore, we gained similar findings for the parameters of \%TWL and \%EWL. Patients in the surgery group lost $15.7 \%$ of the initial weight, while patients in the control group only lost $3.7 \%$ of initial weight within 60 months $(p<0.001)$. The highest \%EWL for patients in the surgery group equalled $69.6 \%$ at 12 months and fluctuated to $48.8 \%$ at the end of follow-up. The \%EWL of non-surgical patients increased slowly, but were significantly lower than that of surgical patients over time $(p<0.001)$. Generally, \%REWL between patients in two groups were not significantly different, except at 3 and 12 months $(\mathrm{p}<0.001)$. (Figure 3f-i)

\section{Comparison between restrictive and bypass surgeries}

Supplemental Figure 6-8 display the disease remission rates and changes of metabolic and weight parameters for surgical patients over time. Overall, the effectiveness of restrictive and bypass surgeries were similar. No significant difference was found in remission rates of hypertension and dyslipidaemia between two different surgical procedures. Of note, patients 
underwent restrictive surgeries had significantly higher attrition-adjusted prevalent and cumulative diabetes remission rates from 24 months onwards. However, patients underwent bypass surgeries had significantly lower LDL-C level until 36 months. Also, bypass surgeries showed better effectiveness in weight reduction within 12 months after surgery. However, it was probably due to the fact that bypass patients had lower baseline BMI than restrictive patients (34.72 kg/m² vs $\left.37.84 \mathrm{~kg} / \mathrm{m}^{2}, \mathrm{p}<0.001\right)$.

\section{Discussion}

To best of our knowledge, the present study is the largest cohort study that evaluated the effectiveness of bariatric surgery among Chinese population using population-based data (1517, 38-40). Also, this study made a comprehensive illustration of metabolic and weight outcomes, including complete and partial remission rates of diabetes, remission rates of hypertension, high LDL-C, low HDL-C, high triglycerides and dyslipidaemia, and improvement of BMI, HbA1c, SBP, DBP, LDL-C, \%TWL, \%EWL, and \%REWL. The definition of disease remission after bariatric surgery varied greatly from one study to another (16, 41-44), impeding direct comparisons among studies. In this study, T2DM, hypertension and dyslipidaemia remission were defined based on the most updated guidelines published by American Diabetes Association, European Society Cardiology and the European Society of Hypertension and AACE/ACE, respectively (33-35), since these guidelines are widely accepted. As suggested by Isaman et al (31), results of existing studies that evaluated effectiveness of bariatric surgery on disease remission varied widely, hence additional reporting on both attrition-adjusted cumulative and prevalent remission rates after bariatric surgery could support in decision making process and making comparison across studies. In the present study, prevalent remission rates, attrition-adjusted prevalent remission rates, cumulative remission rates, and attrition-adjusted cumulative remission rates were all measured in both surgical and matched control groups. Also, we compared the effectiveness of two different types of surgery, the results of which could help bariatric surgeons in choosing a suitable surgery for patients. Combined with above strengths, our findings can reflect the effectiveness of bariatric surgery among obese patients with T2DM and support fair comparison of remission rates among studies. 
The results of this study were in line with the conclusion of previous observational studies and randomised controlled trials (RCTs), which found that bariatric surgery provided substantial and sustainable effects on weight loss, glycaemic control and lipid profile improvement (15, 42, 44-46). However, observations of the present study were at variance with other studies regarding to diabetes remission and weight loss in quantitative aspect and hypertension remission from qualitative perspective.

Though the effects of bariatric surgery on T2DM was confirmed in this study, our results of prevalent complete diabetes remission rates were relatively low when compared with studies focused on either Caucasians or Chinese populations (16, 39, 44, 47). For instance, Swedish Obese Subjects (SOS) study, one of the largest and longest prospective study, found that T2DM recovery rates of Swedish patients with bariatric surgery were $72 \%$ at 2 years (47); but only $26.4 \%$ of surgical patients at 24 months were found to achieve diabetes remission in our study. The quantitative differences in diabetes recovery rates among studies may due to the heterogeneity of remission definition. Actually, many existing studies that discussed the effectiveness of bariatric surgery on T2DM had largely varied T2DM remission criteria, leading to direct comparisons across studies difficult and inappropriate. However, another single-centre observational study in Hong Kong (16), which adopted the same stringent definition of diabetes remission as we did to evaluate the 5-year T2DM remission rates among 65 Chinese patients with LSG, still reported relatively higher T2DM remission rates than us. Apart from sample size, baseline duration of T2DM, fasting glycaemia, HbA1c and waist circumference of included patients were all essential determinants that led to the differences of T2DM remission rates between studies (48).

This study confirmed that surgical patients experienced more improvements of lipid profile, as remission rates of hyperlipidaemia, low HDL-C, high LDL-C and hypertriglyceridemia of surgical patients were significant higher than their non-surgical peers. However, unlike the results reported by Jamal et al (42), mean LDL-C level of surgical patients in the current study did not dropped greatly over the years and was not significantly lower than those of nonsurgical peers. This was because the baseline LDL-C of our surgical cohort was only 2.39 $\mathrm{mmol} / \mathrm{L}$, which was lower than the cut-off of high LDL-C $(\geq 2.59 \mathrm{mmol} / \mathrm{L})$. In the contrast, the 
baseline LDL-C of patients in the mentioned cohort study (42) was $3.50 \mathrm{mmol} / \mathrm{L}$ (135 mg/dL), and thus those patients had more room in LDL-C reduction.

Our study did not support that bariatric surgery was associated with hypertension remission. Reported hypertension remission rate of an observational cohort study was 38.2\% among LGB participates at year 3 post surgery (46), while the prevalent hypertension remission rates in our study did not exceed $6.5 \%$ over the years. One possible explanation of the relatively low hypertension remission rates in our study was that a stringent criterion of hypertension remission was adopted in our study; whereas the definition of hypertension remission of the mentioned cohort study (46) did not require patients to stay hypertension-free for at least one year.

Occurrence of weight regain after bariatric surgery was acknowledged in several studies (49, 50), as well as in the current study. Change patterns of both BMI and \%TWL in our study verified the weight rebound in Hong Kong patients with bariatric surgery. However, though there was weigh rebound, reported \%TWL and \%EWL in a US cohort (50) and a meta-analysis (5) was around $30 \%$ and $60 \%$, respectively, within 5 years. Compared with those studies, both \%TWL and \%EWL of the present study were relatively low, possibly resulting from difference of ethics origin. Actually, both \%EWL and \%REWL of the present study were close to the ones reported by the local study (16). Notably, patients in the control group also experienced weight loss over the years and around 5\% of control patients achieved T2DM remission, although their \%TWL did not exceed 5\% as stated in the previous literature review (49). This may because most of diabetic patients in Hong Kong were enrolled in the Risk Assessment and Management Program (RAMP-DM), which is a territory-wide program launched by HA in 2008 (26). This program, assessed individual risk level and managed patients by multidisciplinary teams, reduced the HbA1c and BMI within 5-year follow-up (26).

Several limitations of the present study should be acknowledged. Firstly, the inclusion criteria of BMI for patients in the surgery and the control groups was slightly different. For surgical patients, we used guideline-recommended BMI cut-off for Asian patients who should consider bariatric surgery (28), while we adopted WHO’s definition of obesity (30) for the control group. 
Despite of the discrepancy, the baseline BMI of both groups were well balanced. Secondly, over half of enrolled surgical patients underwent bariatric surgery after the year of 2013, and they did not achieve 5-year follow-up. Besides, due to the nature of observational database study, clinical parameters were routinely measured for those complying with healthcare followup appointments, and not available for those who defaulted or re-scheduled the appointments. Hence, the results of this study, especially those of 48-60 months, may be biased. Our comparative results between restrictive and bypass surgeries indicated that restrictive surgeries were slightly more preferred for T2DM patients, while bypass surgeries were more effective in LDL-C and weight reduction in first few months after surgery. However, as less than 50\% of initial study population were available for analysis at the end of follow-up, surgeons should be cautious when using these results, especially those of later years. In addition, we limited the follow-up period in this study to 60 months. Ideally, an extensive follow-up period would allow robust data analyses and generate more reliable results. For example, the follow-up duration of the SOS trial (51) was 12-25 years and that of SurgiCal Obesity Treatment Study (SCOTS) (52) will be 10 years or beyond. However, though bariatric surgery had a history of over 60 years, it was firstly introduced to Hong Kong in the early 2000s and only started rising only in recent few years (20). Therefore, studies with larger sample size and longer follow-up duration should be conducted in the future. Lastly, like most comparative studies $(6,18,40)$, this study compared the patients with bariatric surgery with those under usual care. However, comparison of effectiveness between bariatric surgery and conventional therapies, such as lifestyle interventions and medications, is not proper to some extent. This is not only because hypotheses that bariatric surgery is superior to usual care in metabolic parameter improvement and disease remission have been widely confirmed by numerous previous studies (5), but also due to the fact that surgical patients are mainly those who failed first-line therapies (10) and are supposed to be less healthy than non-surgical patients. Besides, though this propensity score-matched study using real-word data reflect the actual clinical aspects, RCTs are actually given the highest level of evidence since they are less biased and have less risk of systematic errors (53). Therefore, a more appropriate design of RCT for the measurement of effectiveness of bariatric surgery versus other relevant therapies still needs further discussion.

In conclusion, among obese T2DM patients, bariatric surgery was associated with increased remission of diabetes and hyperlipidaemia, and reduction in body weight, BMI, HbA1c and LDL-C in 5-year post-surgery. The effectiveness of restrictive and bypass surgeries was similar 
at the end of follow-up, though restrictive surgeries were slightly more effective in T2DM remission.

\section{Acknowledgements}

The authors wish to acknowledge the Central Panel on Administrative Assessment of External Data Requests, Hong Kong Hospital Authority Head Office, for the provision of data, and $\mathrm{Mr}$ Chu Wa Ho and Mr Eric Tang for statistical assistance. Dr. Carlos KH Wong is the guarantor of this study.

\section{Conflict of Interest Statement}

The authors declare that they have no competing interests.

\section{Authors' Contribution Statement}

C.W. constructed the study design. C.W. and T.W. conducted the data analysis. T.W. was responsible for the paper draft and data interpretation. S.W., B.L., E.G., O.W., D.T., D.L. and C.L were responsible for expertise advice and final approval. All authors read and approved the final manuscript.

\section{Ethical approval}

All procedures performed in studies involving human participants were in accordance with the ethical standards of the institutional and/or national research committee and with the 1964 Helsinki declaration and its later amendments or comparable ethical standards. Ethics approval of this study was granted by Institutional Review Board of the University of Hong Kong /Hospital Authority Hong Kong West Cluster (Ref No. UW 16-1018).

\section{Availability of data and material}

The data that support the findings of this study are available from database of the Clinical Management System of the Hospital Authority, Hong Kong but restrictions apply to the 
availability of these data, which were used under license for the current study, and so are not publicly available. Data are however available from the authors upon reasonable request and with permission of Hospital Authority, Hong Kong.

\section{Funding}

This study was funded by Health and Medical Research Fund Research Fellowship Scheme, Food and Health Bureau, HKSAR (Ref No.\#02160087). No funding organization had any role in the design and conduct of the study; collection, management, analysis, and interpretation of the data; and preparation of the manuscript. All other authors have reported that they have no relationships relevant to the contents of this paper to disclose.

\section{List of abbreviations}

American Association of Clinical Endocrinologists and American College of Endocrinology (AACE/ACE)

Body Mass Index (BMI)

Charlson Comorbidity Index (CCI)

Confidence Interval (CI)

Clinical Management System (CMS)

Diastolic Blood Pressure (DBP)

Hospital Authority (HA)

Haemoglobin A1c (HbA1c)

High-Density Lipoprotein Cholesterol (HDL-C)

International Classification of Disease, Ninth Revision, Clinical Modification (ICD-9-CM)

International Classification of Primary Care version 2 (ICPC-2)

International Federation for the Surgery of Obesity and Metabolic Disorders - Asia Pacific Chapter (IFSO-APC)

Laparoscopic Adjustable Gastric Banding (LAGB) 
Low-Density Lipoprotein Cholesterol (LDL-C)

Laparoscopic Gastric Bypass (LGB)

Laparoscopic Sleeve Gastrectomy (LSG)

Laparoscopic Sleeve Gastrectomy with Duodenojejunal Bypass (LSG/DJB)

Multiple imputation by chained equations (MICE)

Percentage of Excess Weight Loss (\%EWL)

Percentage of Rebound in Excess Weight Loss (\%REWL)

Percentage of Total Weight Loss (\%TWL)

Randomised Controlled Trial (RCT)

Systolic Blood Pressure (SBP)

SurgiCal Obesity Treatment Study (SCOTS)

Standard deviation (SD)

Swedish Obese Subjects (SOS)

Type 2 diabetes mellitus (T2DM)

World Health Organisation (WHO) 


\section{References}

1. Dixon JB, Chuang LM, Chong K, Chen SC, Lambert GW, Straznicky NE, et al. Predicting the glycemic response to gastric bypass surgery in patients with type 2 diabetes. Diabetes Care. 2013;36(1):20-6.

2. Brolin RE. Bariatric surgery and long-term control of morbid obesity. JAMA. 2002;288(22):2793-6.

3. Simonson DC, Halperin F, Foster K, Vernon A, Goldfine AB. Clinical and PatientCentered Outcomes in Obese Patients With Type 2 Diabetes 3 Years After Randomization to Roux-en-Y Gastric Bypass Surgery Versus Intensive Lifestyle Management: The SLIMMT2D Study. Diabetes Care. 2018;41(4):670-9.

4. Bennett JMH, Mehta S, Rhodes M. Surgery for morbid obesity. Postgrad Med J. 2007;83(975):8.

5. Buchwald H, Avidor Y, Braunwald E, Jensen MD, Pories W, Fahrbach K, et al. Bariatric Surgery: A Systematic Review and Meta-analysis. JAMA. 2004;292(14):1724-37.

6. Gloy VL, Briel M, Bhatt DL, Kashyap SR, Schauer PR, Mingrone G, et al. Bariatric surgery versus non-surgical treatment for obesity: a systematic review and meta-analysis of randomised controlled trials. BMJ: British Medical Journal. 2013;347(7931):11-.

7. Toh BC, Chan WH, Eng AKH, Lim EKW, Lim CH, Tham KW, et al. Five-year longterm clinical outcome after bariatric metabolic surgery: A multi-ethnic Asian population in Singapore. Diabetes Obes Metab. 2018;20(7):1762-5.

8. Puzziferri N, Roshek TB, Mayo HG, Gallagher R, Belle SH, Livingston EH. Long-term Follow-up After Bariatric Surgery: A Systematic Review. JAMA. 2014;312(9):934-42.

9. Sjöström L, Peltonen M, Jacobson P, et al. Association of bariatric surgery with longterm remission of type 2 diabetes and with microvascular and macrovascular complications. JAMA. 2014;311(22):2297-304.

10. American Diabetes A. 8. Obesity Management for the Treatment of Type 2 Diabetes: Standards of Medical Care in Diabetes-2019. Diabetes Care. 2019;42(Suppl 1):S81-S9.

11. Garvey W, Mechanick J, Brett E, Garber A, Hurley DL, Jastreboff A, et al. American Association of Clinical Endocrinologists and American College of Endocrinology Comprehensive Clinical Practice Guidelines for Medical Care of Patients with Obesity. Endocr Pract. 2016;22(s3):1-203.

12. Jensen DM, Ryan HD, Apovian MC, Ard DJ, Comuzzie GA, Donato AK, et al. 2013 AHA/ACC/TOS Guideline for the Management of Overweight and Obesity in Adults: A Report of the American College of Cardiology/American Heart Association Task Force on Practice Guidelines and The Obesity Society. Circulation. 2014;129(25_suppl_2 Suppl 1):S102-S38.

13. Angrisani L, Santonicola A, Iovino P, Formisano G, Buchwald H, Scopinaro N. Bariatric Surgery Worldwide 2013. OBES SURG. 2015;25(10):1822-32.

14. Huang C-K, Shabbir A, Lo C-H, Tai C-M, Chen Y-S, Houng J-Y. Laparoscopic Rouxen-Y Gastric Bypass for the Treatment of Type II Diabetes Mellitus in Chinese Patients with Body Mass Index of 25-35. OBES SURG. 2011;21(9):1344-9.

15. Zhang Y, Zhao H, Cao Z, Sun X, Zhang C, Cai W, et al. A Randomized Clinical Trial of Laparoscopic Roux-en-Y Gastric Bypass and Sleeve Gastrectomy for the Treatment of Morbid Obesity in China: a 5-Year Outcome. OBES SURG. 2014;24(10):1617-24.

16. Liu S, Wong S, Lam C, Yung M, Kong A, Ng E. Long-term Results on Weight Loss and Diabetes Remission after Laparoscopic Sleeve Gastrectomy for A Morbidly Obese Chinese Population. OBES SURG. 2015;25(10):1901-8. 
17. Zeng T, Cai Y, Chen L. The Effectiveness of Bariatric Surgery for Chinese Obesity in 2 Years: A Meta-Analysis and Systematic Review. Journal of Investigative Surgery. 2017;30(5):332-41.

18. Heo Y-S, Park J-M, Kim Y-J, Kim S-M, Park D-J, Lee S-K, et al. Bariatric surgery versus conventional therapy in obese Korea patients: a multicenter retrospective cohort study. Journal of the Korean Surgical Society. 2012;83(6):335-42.

19. Moon Han S, Kim W, Oh J. Results of Laparoscopic Sleeve Gastrectomy (LSG) at 1 Year in Morbidly Obese Korean Patients. OBES SURG. 2005;15(10):1469-75.

20. Wong SKH, Kong APS, Mui WLM, So WY, Tsung BYS, Yau PYP, et al. Laparoscopic bariatric surgery: a five-year review. Hong Kong medical journal = Xianggang yi xue za zhi. 2009;15(2):100.

21. Rössner S, Sjöström L, Noack R, Meinders AE, Noseda G. Weight loss, weight maintenance, and improved cardiovascular risk factors after 2 years treatment with orlistat for obesity. European Orlistat Obesity Study Group. Obesity research. 2000;8(1):49.

22. Trastulli S, Desiderio J, Guarino S, Cirocchi R, Scalercio V, Noya G, et al. Laparoscopic sleeve gastrectomy compared with other bariatric surgical procedures: a systematic review of randomized trials. Surg Obes Relat Dis. 2013;9(5):816-29.

23. Kosanovic R, Romero RJ, Donkor C, Sarasua A, Rabaza JR, Gonzalez AM. A comparative retrospective study of robotic sleeve gastrectomy vs robotic gastric bypass. Int $\mathrm{J}$ Med Robot. 2015;11(3):275-83.

24. Du X, Zhang SQ, Zhou HX, Li X, Zhang XJ, Zhou ZG, et al. Laparoscopic sleeve gastrectomy versus Roux-en-Y gastric bypass for morbid obesity: a 1:1 matched cohort study in a Chinese population. Oncotarget. 2016;7(46):76308-15.

25. Aragon-Alonso A, Gomez MF, Sanchez JRC, de AMDR, Bernal MDF, Espallardo MAC, et al. Comparative study between laparoscopic gastric bypass and laparoscopic sleeve gastrectomy. Endocrine Abstracts. 2017;49:EP708.

26. Wan EYF, Fung CSC, Jiao FF, Yu EYT, Chin WY, Fong DYT, et al. Five-Year Effectiveness of the Multidisciplinary Risk Assessment and Management ProgrammeDiabetes Mellitus (RAMP-DM) on Diabetes-Related Complications and Health Service UsesA Population-Based and Propensity-Matched Cohort Study. Diabetes Care. 2018;41(1):49-59. 27. Wong CKH, Tong T, Cheng GHL, Tang EHM, Thokala P, Tse ETY, et al. Direct medical costs in the preceding, event and subsequent years of a first severe hypoglycaemia episode requiring hospitalization: A population-based cohort study. Diabetes Obes Metab. 2019;21(6):1330-9.

28. Kasama K, Mui W, Lee W, Lakdawala M, Naitoh T, Seki Y, et al. IFSO-APC Consensus Statements 2011. OBES SURG. 2012;22(5):677-84.

29. Rubino F, Nathan DM, Eckel RH, Schauer PR, Alberti KGMM, Zimmet PZ, et al. Metabolic Surgery in the Treatment Algorithm for Type 2 Diabetes: A Joint Statement by International Diabetes Organizations. Diabetes Care. 2016;39(6):861.

30. World Health Organization. Obesity and Overweight [Internet]. 2018 [cited 2018 November 13]. Available from: http://www.who.int/news-room/fact-sheets/detail/obesityand-overweight.

31. Isaman DJM, Rothberg AE, Herman WH. Reconciliation of type 2 diabetes remission rates in studies of Roux-en-Y gastric bypass. Diabetes Care. 2016;39(12):2247-53.

32. American Diabetes A. 6. Glycemic Targets: Standards of Medical Care in Diabetes2019. Diabetes Care. 2019;42(Suppl 1):S61-S70.

33. Buse JB, Caprio S, Cefalu WT, Ceriello A, Del Prato S, Inzucchi SE, et al. How do we define cure of diabetes? Diabetes care. 2009;32(11):2133. 
34. Williams B, Mancia G, Spiering W, Agabiti Rosei E, Azizi M, Burnier M, et al. 2018 ESC/ESH Guidelines for the management of arterial hypertension. Eur Heart J. 2018;39(33):3021-104.

35. Bush M, Zangeneh F. American Association of Clinical Endocrinologists and American College of Endocrinology Guidelines for Management of Dyslipidemia and Prevention of Cardiovascular Disease. Endocrine Practice. 2017;23(Suppl 2):1-87.

36. Royston P, White IR. Multiple Imputation by Chained Equations (MICE): Implementation in Stata. Journal of Statistical Software. 2011;45(4).

37. White I, Royston P, Wood A. Multiple imputation using chained equations: Issues and guidance for practice. Statistics in Medicine. 2011;30(4):377.

38. Hans PK, Guan W, Lin S, Liang H. Long-term outcome of laparoscopic sleeve gastrectomy from a single center in mainland China. Asian Journal of Surgery. 2018;41(3):28590.

39. Wang X, Chang X-S, Gao L, Zheng C-Z, Zhao X, Yin K, et al. Effectiveness of laparoscopic sleeve gastrectomy for weight loss and obesity-associated co-morbidities: a 3year outcome from Mainland Chinese patients. Surgery for Obesity and Related Diseases. 2016;12(7):1305-11.

40. Feng W, Yin T, Chu X, Shan X, Jiang C, Wang Y, et al. Metabolic effects and safety of Roux-en-Y gastric bypass surgery vs. conventional medication in obese Chinese patients with type 2 diabetes. Diabetes Metab Res Rev. 2019;35(5):e3138.

41. Neff K, Baud G, Raverdy V, Caiazzo R, Verkindt H, Noel C, et al. Renal Function and Remission of Hypertension After Bariatric Surgery: a 5-Year Prospective Cohort Study. OBES SURG. 2017;27(3):613-9.

42. Jamal M, Wegner R, Heitshusen D, Liao J, Samuel I. Resolution of hyperlipidemia follows surgical weight loss in patients undergoing Roux-en-Y gastric bypass surgery: a 6-year analysis of data. Surg Obes Relat Dis. 2011;7(4):473-9.

43. Eliasson B, Liakopoulos V, Franzen S, Näslund I, Svensson A-M, Ottosson J, et al. Cardiovascular disease and mortality in patients with type 2 diabetes after bariatric surgery in Sweden:: A nationwide, matched, observational cohort study. Lancet Diabetes And Endocrinology. 2015;3(11):847-54.

44. Purnell JQ, Selzer F, Wahed AS, Pender J, Pories W, Pomp A, et al. Type 2 Diabetes Remission Rates After Laparoscopic Gastric Bypass and Gastric Banding: Results of the Longitudinal Assessment of Bariatric Surgery Study. Diabetes Care. 2016;39(7):1101-7.

45. Benetti A, Del Puppo M, Crosignani A, Veronelli A, Masci E, Frige F, et al. Cholesterol metabolism after bariatric surgery in grade 3 obesity: differences between malabsorptive and restrictive procedures. Diabetes Care. 2013;36(6):1443-7.

46. Courcoulas AP, Christian NJ, Belle SH, et al. Weight change and health outcomes at 3 years after bariatric surgery among individuals with severe obesity. JAMA. 2013;310(22):2416-25.

47. Sjostrom L, Lindroos AK, Peltonen M, Torgerson J, Bouchard C, Carlsson B, et al. Lifestyle, diabetes, and cardiovascular risk factors 10 years after bariatric surgery. $\mathrm{N}$ Engl $\mathrm{J}$ Med. 2004;351(26):2683-93.

48. Panunzi S, Carlsson L, De Gaetano A, Peltonen M, Rice T, Sjostrom L, et al. Determinants of Diabetes Remission and Glycemic Control After Bariatric Surgery. Diabetes Care. 2016;39(1):166-74.

49. Schauer PR, Mingrone G, Ikramuddin S, Wolfe B. Clinical Outcomes of Metabolic Surgery: Efficacy of Glycemic Control, Weight Loss, and Remission of Diabetes. Diabetes Care. 2016;39(6):902-11. 
50. Maciejewski ML, Arterburn DE, Van Scoyoc L, Smith VA, Yancy WS, Jr., Weidenbacher HJ, et al. Bariatric Surgery and Long-term Durability of Weight Loss. JAMA Surg. 2016;151(11):1046-55.

51. Sjöström L. Review of the key results from the Swedish Obese Subjects ( SOS ) trial a prospective controlled intervention study of bariatric surgery. 2013. p. 219-34.

52. Logue J, Stewart S, Munro J, Bruce J, Grieve E, Lean M, et al. SurgiCal Obesity Treatment Study (SCOTS): protocol for a national prospective cohort study of patients undergoing bariatric surgery in Scotland. BMJ Open. 2015;5(5):e008106.

53. Burns PB, Rohrich RJ, Chung KC. The levels of evidence and their role in evidencebased medicine. Plast Reconstr Surg. 2011;128(1):305-10. 


\section{Figure legend}

Figure 1: Prevalent remission rates of patients in the surgery and control group, a) diabetes complete; b) diabetes complete or partial; c) hypertension; d) dyslipidaemia; e) Attritionadjusted prevalent diabetes complete; f) Attrition-adjusted prevalent diabetes partial; g) Attrition-adjusted prevalent hypertension; and h) Attrition-adjusted prevalent dyslipidaemia Figure 2: Cumulative remission rates of patients in the surgery and control group, a) diabetes complete; b) diabetes complete or partial; c) hypertension; d) dyslipidaemia; e) Attritionadjusted prevalent diabetes complete; f) Attrition-adjusted prevalent diabetes partial; g) Attrition-adjusted prevalent hypertension; and h) Attrition-adjusted prevalent dyslipidaemia Figure 3: Changes in weight loss and metabolic outcomes in the surgery and control group, a) HbA1c, b) HbA1c<7\%, c) systolic blood pressure, d) diastolic blood pressure, e) low-density lipoprotein cholesterol, f) body mass index, g) percentage of total weight loss, h) percentage of excess weight loss; and i) percentage of rebound in excess weight loss 


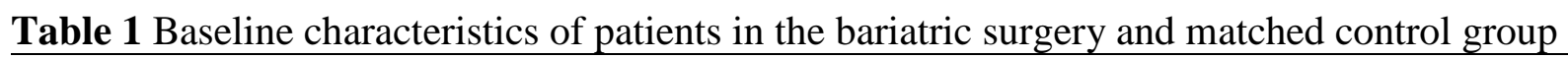

\begin{tabular}{|c|c|c|c|c|c|}
\hline \multirow[b]{2}{*}{ Characteristics } & \multirow{2}{*}{$\begin{array}{c}\text { Before matching } \\
\begin{array}{c}\text { Bariatric surgery patients } \\
(\mathrm{n}=422)\end{array}\end{array}$} & \multicolumn{4}{|c|}{$\begin{array}{l}\text { After 1-to-5 propensity score matching } \\
\end{array}$} \\
\hline & & $\begin{array}{l}\text { Bariatric surgery patients } \\
(\mathrm{n}=401)\end{array}$ & $\begin{array}{l}\text { Matched control patients } \\
(\mathrm{n}=1894)\end{array}$ & $\mathrm{p}$-value & SMD \\
\hline \multicolumn{6}{|l|}{ Socio-demographic } \\
\hline Female, n (\%) & $210(49.8)$ & $201(50.1)$ & $966(47.6)$ & 0.353 & 0.051 \\
\hline Mean age (SD), year & 53.21 (13.39) & 53.36 (13.29) & $53.28(13.70)$ & 0.916 & 0.006 \\
\hline \multicolumn{6}{|l|}{ Clinical parameters } \\
\hline Mean BMI (SD), kg/m² & $37.14(5.42)$ & $36.81(5.09)$ & $36.31(6.13)$ & 0.121 & 0.090 \\
\hline Mean weight (SD), kg & 100.53 (18.94) & 99.62 (18.09) & $93.90(18.51)$ & $<0.001^{*}$ & 0.313 \\
\hline Mean height (SD), m & $1.64(0.09)$ & $1.64(0.09)$ & $1.61(0.11)$ & $<0.001^{*}$ & 0.345 \\
\hline Mean waist circumference, $\mathrm{cm}$ & $113.60(12.33)$ & $113.17(12.03)$ & $109.73(12.72)$ & $<0.001^{*}$ & 0.278 \\
\hline Mean HbA1c, \% & $7.65(1.54)$ & $7.66(1.51)$ & $7.56(1.77)$ & 0.262 & 0.065 \\
\hline Mean SBP (SD), mmHg & $134.30(16.89)$ & 134.66 (16.76) & $134.00(16.69)$ & 0.520 & 0.040 \\
\hline Mean DBP (SD), mmHg & $77.26(10.33)$ & $77.50(10.37)$ & $77.35(10.32)$ & 0.809 & 0.015 \\
\hline Mean total cholesterol (SD), mmol/L & $4.32(0.93)$ & $4.31(0.93)$ & $4.27(0.92)$ & 0.362 & 0.050 \\
\hline Mean HDL-C (SD), mmol/L & $1.08(0.30)$ & $1.09(0.29)$ & $1.08(0.27)$ & 0.536 & 0.033 \\
\hline Mean TC/HDL-C ratio (SD) & $4.24(1.53)$ & $4.20(1.31)$ & 4.14 (1.18) & 0.364 & 0.049 \\
\hline Mean LDL-C (SD), mmol/L & $2.40(0.81)$ & $2.39(0.80)$ & $2.35(0.75)$ & 0.297 & 0.057 \\
\hline Mean triglyceride (SD), mmol/L & $1.93(1.48)$ & $1.88(1.21)$ & $1.90(1.33)$ & 0.842 & 0.011 \\
\hline Mean fasting glucose (SD), mmol/L & $7.96(2.86)$ & $8.01(2.86)$ & $7.80(2.78)$ & 0.181 & 0.074 \\
\hline Mean serum creatinine (SD), umol/l & $83.51(66.05)$ & $84.31(67.59)$ & 83.96 (54.63) & 0.910 & 0.006 \\
\hline Mean eGFR (SD), ml/min/1.73m² & $93.02(30.95)$ & 92.35 (31.03) & $90.82(30.14)$ & 0.359 & 0.050 \\
\hline Mean duration of DM (SD), year & $4.02(3.14)$ & $4.12(3.14)$ & $4.36(2.84)$ & 0.136 & 0.079 \\
\hline Insulin used, n (\%) & $83(19.7)$ & $78(19.5)$ & $362(19.1)$ & 0.876 & 0.009 \\
\hline Oral anti-diabetic drugs ever used, n (\%) & $235(55.7)$ & $231(57.6)$ & $1127(59.5)$ & 0.495 & 0.037 \\
\hline \multicolumn{6}{|l|}{ Comorbidities } \\
\hline History of hypertension, n (\%) & $334(79.2)$ & 319 (79.6) & 1464 (77.3) & 0.336 & 0.054 \\
\hline Anti-hypertensive drugs ever used, n (\%) & $326(77.3)$ & $313(78.1)$ & $1470(77.6)$ & 0.829 & 0.012 \\
\hline History of hyperlipidaemia, n (\%) & $218(51.7)$ & $205(51.1)$ & $960(50.7)$ & 0.874 & 0.009 \\
\hline Lipid lowering agents ever used, n (\%) & $225(53.3)$ & $214(53.4)$ & 1059 (55.9) & 0.351 & 0.051 \\
\hline History of mental health problems, n (\%) & $22(5.2)$ & $21(5.2)$ & $112(5.9)$ & 0.598 & 0.029 \\
\hline History of obstructive sleep apnoea, n (\%) & $235(55.7)$ & $220(54.9)$ & $1040(54.9)$ & 0.986 & 0.001 \\
\hline History of gall bladder disease, n (\%) & $36(8.5)$ & $34(8.5)$ & $140(7.4)$ & 0.455 & 0.040 \\
\hline $\begin{array}{l}\text { History of musculoskeletal and chronic orthopaedic } \\
\text { disorders, } n(\%)\end{array}$ & $103(24.4)$ & $98(24.4)$ & $481(25.4)$ & 0.689 & 0.022 \\
\hline Mean Charlson comorbidity index (SD) & $3.68(1.92)$ & $3.70(1.95)$ & $3.58(1.90)$ & 0.226 & 0.066 \\
\hline
\end{tabular}

Notes:

Abbreviations: SMD = Standardised Mean Difference; SD = Standard Deviation; BMI = Body Mass Index; SBP = Systolic Blood Pressure; DBP = Diastolic Blood Pressure; HDL-C = Highdensity Lipoprotein Cholesterol; TC = Total Cholesterol; LDL-C = Low-density Lipoprotein Cholesterol; eGFR = estimated glomerular filtration rate; DM = Diabetes Mellitus *Significant difference ( $\mathrm{p}$-value $<0.05$ ) detected by independent $\mathrm{t}$ tests or by chi square tests.

${ }^{\text {I }} \mathrm{SMD}<0.100$ indicates the balance of baseline covariates. 


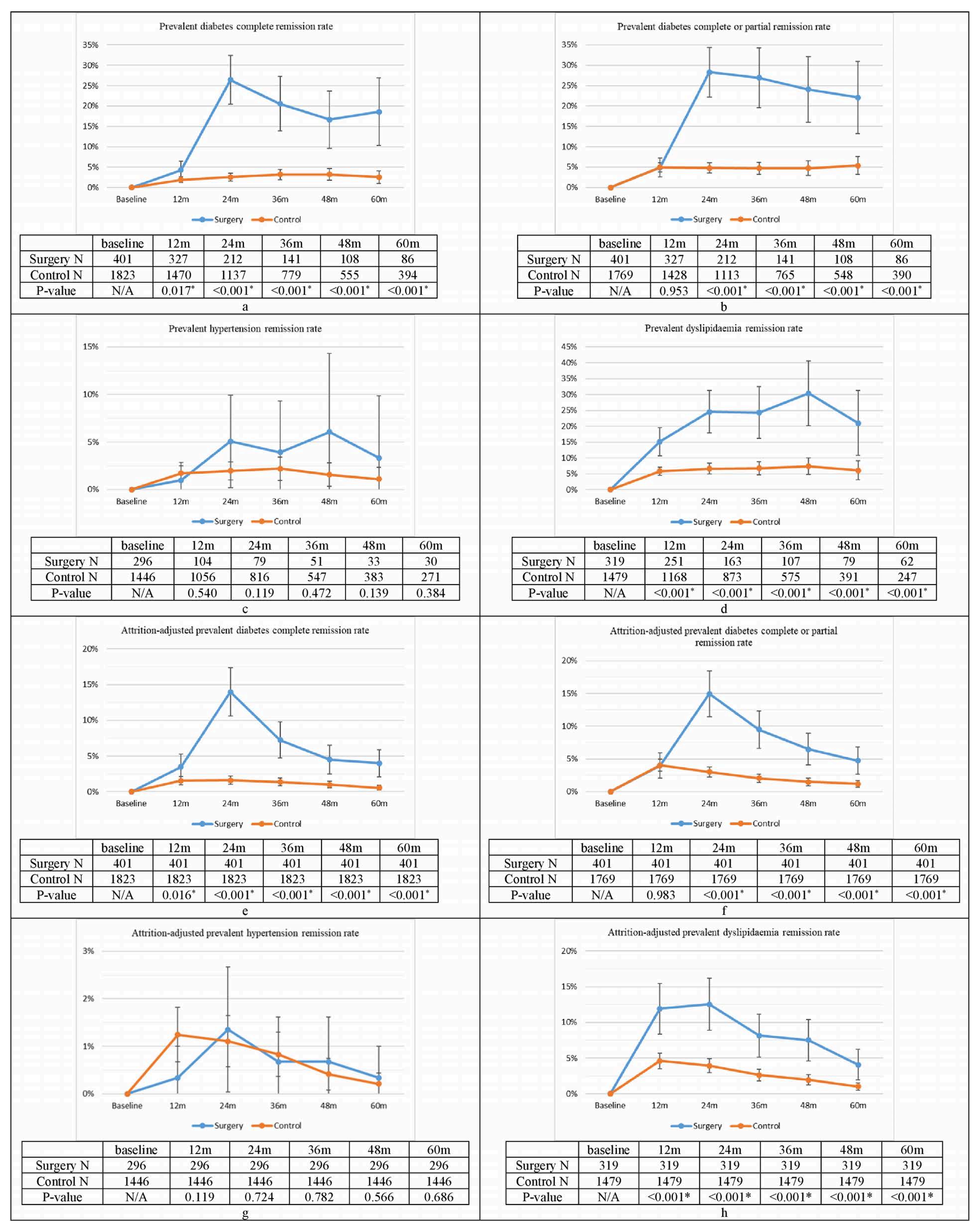




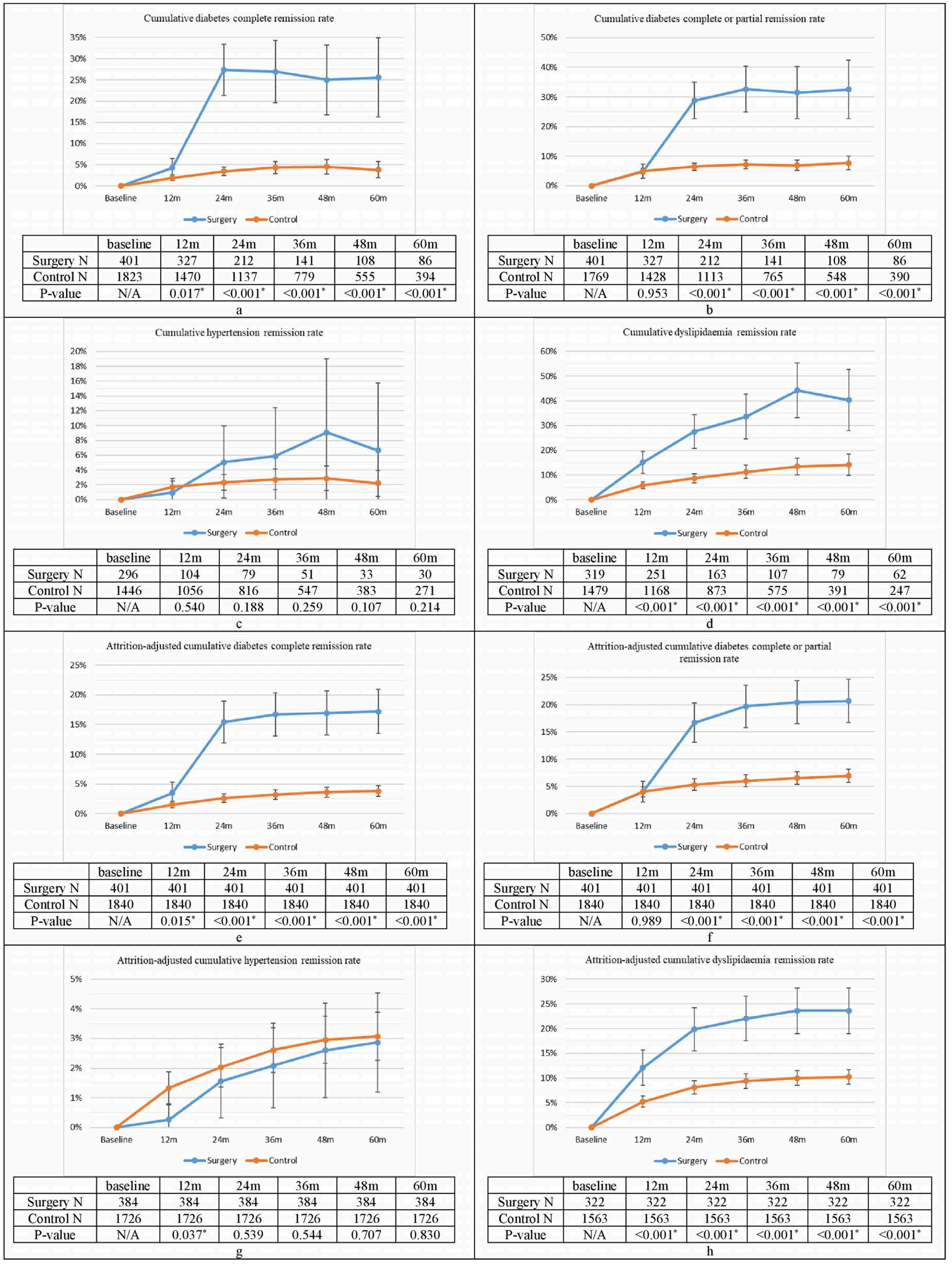


